\title{
RESEARCH
}

\section{Rapid tranquillisation: practice in Zambia, before and after training}

\author{
Mahesh Jayaram, ${ }^{1}$ Ranga Rattehalli, ${ }^{2}$ Lindsay Moran, ${ }^{3}$ John Mwanza, ${ }^{4}$ \\ Paul Banda ${ }^{5}$ and Clive Adams ${ }^{6}$
}

${ }^{1}$ Consultant Psychiatrist, Aire Court, Lingwell Grove, Leeds, UK, email mahesh.jayaram@nhs.net

${ }^{2}$ Consultant Psychiatrist, Newsam Centre, Seacroft Hospital, Leeds, UK, email rdrattehalli@hotmail. com

${ }^{3}$ General Practitioner, The Gables Surgery, Leeds, UK, ema lindsaymoran@doctors.org.uk

${ }^{4}$ Chainama Hills College

Hospital, Lusaka, Zambia, email mjmwanza24@yahoo.com ${ }^{5}$ Chainama Hills College Hospital, Lusaka, Zambia, email paulbanda01@yahoo.com ${ }^{6}$ Professor of Psychiatry, University of Nottingham, Vottingham, UK, email clive. dams@nottingham.ac.uk

We wish to thank all the participants in this survey. We also wish to thank THET and Leeds and York Partnerships NHS Foundation Trust, which funded transportation costs for some of our medical members visiting Zambia.
The evidence base for rapid tranquillisation is small in higher-income countries but is even smaller in sub-Saharan Africa. We initiated the first ever survey on the use of rapid tranquillisation in Zambia in 2009; a further survey was then done in 2010, after a programme of teaching and training. It demonstrated an overall improvement in clinical practice, safety, awareness and use of medications within therapeutic doses. It also led to a reduction in inappropriate use of medications. These improvements in practice occurred within a short time span and with minimal effort. Further international collaborative partnerships are required to build stronger mental health infrastructure in Zambia.

The provision of mental health services in lowand middle-income countries is varied and is often particularly poorly resourced (Saxena et al, 2005). Sub-Saharan Africa has suffered from political strife and financial instability. The region carries $24 \%$ of the world's disease burden but has only $3 \%$ of health workers and less than $1 \%$ of the world's financial resources (World Health Organization, 2006a). In the midst of all this, governments in sub-Saharan Africa perceive funding for mental health to be a luxury. When conditions such as HIV/AIDS, cerebral malaria and perinatal morbidity are such major causes of low average life expectancy, funding the care of an often heavily stigmatised group of people with long-standing depression, dementia and other serious and enduring mental illnesses may not seem attractive. This has led to chronic underinvestment in mental health services, with consequent overcrowding, erosion of morale among staff and derelict infrastructure. Locked wards, infringements of human rights and patient autonomy, and a skeleton staff of overworked and undertrained personnel are common problems associated with mental health services in Zambia. This is compounded by inadequate provision of food for patients and the limited availability of drugs. These only begin to highlight the issues encountered by patients and staff at the Chainama Hills College Hospital, a tertiary mental health hospital in Lusaka, Zambia.

Episodes of acute agitation or behavioural disturbances due to psychiatric illnesses may be a feature of presentations in out-patient clinics, in-patient wards and emergency room settings, as well as in police custody. Acute agitation can occur in up to $10 \%$ of psychiatric emergencies. Common causes include psychotic symptoms (Sachdev, 1996), substance misuse, severe anxiety states (Atakan \& Davies, 1997) and delirium. It is often members of the patient's family who become victims of violence and, not uncommonly, medical and nursing staff. Patients themselves or other patients are also at risk (Bourget et al, 2002).

Clinicians have to decide quickly what intervention is the safest in managing these episodes. Calming the patient down rather than sedation is often thought to be desirable. Guidelines predominantly drawn up in higher-income countries recommend the use of non-pharmacological alternatives as the first line of management, although this may be an impractical approach in lowerincome countries, where resources are stretched and the number of patients often very large (Andrade, 2007). In these crowded circumstances disturbance is common and rapid tranquillisation is one approach used to solve the problem.

Leeds and York Partnership NHS Foundation Trust and Chainama Hills College Hospital set up a collaborative partnership. The first stage of our collaboration was to survey the frequency and circumstances of these difficult situations, and the drugs used.

\section{Method}

The protocol for this work was discussed and approved by the local drugs and therapeutics committee, which was the only committee to sit regularly at Chainama Hills College Hospital. All patients attending the out-patient clinic for a period of 5 consecutive weeks (beginning June 2009) requiring rapid tranquillisation were included. A simple survey questionnaire was completed by trainee clinical officers and medical students not involved in the clinical decision-making or the administrative aspects of patient care.

Following the period of data collection, results were disseminated and discussions took place around best practice. A local protocol was drawn up using available resources. An interactive training session was conducted for all the staff in the hospital aimed at improving knowledge and building skills in the area of rapid tranquillisation. Following this, a repeat survey over a shorter period but otherwise using the same methods was undertaken in March 2010 to see whether there had been any improvements in practice.

\section{Results and discussion}

During the first survey period, 105 patients required rapid tranquillisation (around $8 \%$ of the 
Table 1

Medications used for rapid tranquillisation

\begin{tabular}{|c|c|c|c|c|c|c|}
\hline \multirow{2}{*}{ Drug group } & \multicolumn{2}{|c|}{ Number $(\%)$ of patients } & \multirow{2}{*}{ Route } & \multirow{2}{*}{ Drug } & \multicolumn{2}{|c|}{ Mean dose $(\mathrm{mg})$} \\
\hline & $2009(n=105)$ & $2010(n=16)$ & & & 2009 & 2010 \\
\hline \multirow{8}{*}{ Antipsychotic } & \multirow{8}{*}{$84(80 \%)$} & \multirow{8}{*}{$13(81 \%)$} & i.m. & Haloperidol & 9.6 & 10 \\
\hline & & & Oral & Haloperidol & 6.0 & 5 \\
\hline & & & Oral & Chlorpromazine & 85.5 & 100 \\
\hline & & & Oral & Fluphenazine & 25 & NA \\
\hline & & & i.v. & Haloperidol & 10.9 & 10 \\
\hline & & & Oral & Trifluoperazine & 5.2 & 5 \\
\hline & & & i.m. & Chlorpromazine & 55.5 & NA \\
\hline & & & Oral & Risperidone & 2 & NA \\
\hline Anticholinergic & $46(44 \%)$ & $4(25 \%)$ & Oral & Trihexyphenidyl & 5 & 5 \\
\hline \multirow{3}{*}{$\begin{array}{l}\text { Mood stabiliser } \\
\text { (including } \\
\text { antidepressants) }\end{array}$} & \multirow{3}{*}{$10(10 \%)$} & \multirow{3}{*}{$5(31 \%)$} & Oral & Carbamazepine & 200 & 200 \\
\hline & & & Oral & Amitriptyline & 37.5 & NA \\
\hline & & & Oral & Sodium valproate & 100 & 100 \\
\hline \multirow{3}{*}{ Benzodiazepine } & \multirow{3}{*}{$53(50 \%)$} & \multirow{3}{*}{$10(63 \%)$} & i.m. & Diazepam & 16.2 & 10 \\
\hline & & & i.v. & Diazepam & 13.8 & 5 \\
\hline & & & Oral & Diazepam & 4.6 & 10 \\
\hline Antihistamine & $3(3 \%)$ & $3(19 \%)$ & Oral & Promethazine & 25 & 25 \\
\hline \multirow{2}{*}{ Antiepileptic } & \multirow{2}{*}{$11(10 \%)$} & \multirow{2}{*}{$7(44 \%)$} & Oral & Phenobarbitone & 30 & 30 \\
\hline & & & i.m. & Phenobarbitone & 200 & 200 \\
\hline \multirow{2}{*}{ Others } & \multirow{2}{*}{$9(9 \%)$} & \multirow{2}{*}{$0(0 \%)$} & Oral & Vitamin B & NA & NA \\
\hline & & & Oral & Cloxacillin & 500 & NA \\
\hline
\end{tabular}

i.m., intramuscular; i.v., intravenous; NA, not available (no use recorded).

Table 2

Diagnoses of patients receiving rapid tranquillisation

\begin{tabular}{|l|c|c|}
\hline \multirow{2}{*}{ Diagnosis } & \multicolumn{2}{|c|}{$\%$} \\
\cline { 2 - 3 } & 2009 & 2010 \\
\hline Non-affective psychosis & 37 & 24 \\
\hline Alcohol/substance misuse & 25 & 12 \\
\hline Affective disorder & 4 & 0 \\
\hline Acute confusional state & 5 & 6 \\
\hline Dementing illnesses & 3 & 6 \\
\hline Not mentioned & 9 & 0 \\
\hline Encephalitis & 1 & 0 \\
\hline Epilepsy & 13 & 35 \\
\hline Intellectual disability & 2 & 0 \\
\hline Post-traumatic stress disorder & 2 & 0 \\
\hline Personality disorder & 0 & 17 \\
\hline
\end{tabular}

total number of patients attending the hospital casualty department). No patient was lost to follow-up. About two-thirds were men (68\%); the average age was 32 years (s.d. 14.9, range 2.5-83 years; note that local practice is to bring even young children with epilepsy to psychiatric hospital, which could explain the lower end of this range). The degree of agitation as rated by clinicians (based on their observation of the patient's behaviour and their general clinical impression) was categorised as mild in 53\% of cases, moderate in 30\% and severe in $16 \%$. Numerous combinations of medications and means of delivery were used; $87 \%$ of patients received either intramuscular or intravenous preparations (in some instances both) and 13\% only oral preparations.

In light of the discovery of the varying rapid tranquillisation practices, and a lack of formal teaching, we piloted an interactive training programme for all the staff in the hospital working with people presenting with a mental illness. Two such training workshops were held over a 2-week period so that all staff could attend. Each 3-hour training session was facilitated by a consultant general adult psychiatrist and a mental health nurse visiting from the UK. Each was divided into the following five sections:

1. an interactive discussion on staff and trainee psychiatrists' experiences of dealing with psychiatric emergencies needing rapid tranquillisation (in these discussions, a sense of uncertainty was a prominent theme)

2. an overview of the available guidelines on rapid tranquillisation, including the principles of rapid tranquillisation and the suitability of locally available drugs, their dosage and route of administration

3. a practical approach to the key elements of control and restraint, and the importance of deescalation and its techniques

4. a discussion of the study findings and the available evidence in this area and how this evidence applies to the patients seen in that hospital

5. real-life case studies and case vignettes suitable for this training, which were discussed in smaller groups to build their confidence in handling psychiatric emergencies.

The second survey, in 2010, was conducted over 1 week only, due to resource limitations and lack of suitable professionals to continue data collection for 5 weeks. Otherwise, the same method of data collection was used and no loss to follow-up was ensured. Sixteen patients required rapid tranquillisation (around 15\% of the total attendances at casualty). Again, around two-thirds $(60 \%)$ were men and the average age was 30.2 years. Of those requiring rapid tranquillisation, about $31 \%$ were rated as being in a mild state of agitation, $44 \%$ a moderate state and $16 \%$ a severely agitated state, based on the general clinical impression of the clinician.

Table 1 presents summary statistics for the two surveys. Table 2 shows the use of rapid tranquillisation according to the diagnosis of the patients. In $2009,27 \%$ of the patients subject to rapid tranquillisation had been brought in under restraint to the hospital. These restraints took the form of shackles, ropes and physical restraint by a member of family. Most (61\%) of those admitted in this manner continued to need restraint until after rapid tranquillisation. Overall, 25\% of those who received rapid tranquillisation had to be restrained. Nearly $20 \%$ of patients required repeat medications for sedation. In 2010, 30\% were brought in under restraint, of whom $50 \%$ required restraint for rapid tranquillisation.

Zambia and the UK are collaborating to improve psychiatric training - although more needs to be done. The work done by our teams 
in terms of teaching and training appears to have had an impact in a short time. Discussions around the value of rapid tranquillisation, in particular the safe and effective use of available resources, appears to have been incorporated into some areas of local practice. For example, during the second survey, it was clear that more people with psychosis were getting medication, the doses of medications used were more in line with available evidence of safe use and fewer people with intellectual disabilities or affective disorders were rapidly tranquillised. In summary, the training seemed to have made a difference in the following areas:

- clearer recognition of when rapid tranquillisation was needed

- judicious use of medications at appropriate doses, leading to better availability of medications for those who needed them most

- de-escalation techniques used more effectively

- drug prescribing closer to recommended guidelines, in keeping with resource limitations.

\section{Conclusions}

There are few surveys worldwide of what goes on in this unattractive end of healthcare. This study highlighted variations in treatment, although in fact practice was similar to that in other places (Pilowsky et al, 1992). More widely, the use of rapid tranquillisation may be becoming more consistent and rational than it was in the past (Huf et al, 2002a). Inconsistency thrives when good evidence is lacking. When the evidence base is small or biased, guidance can be contradictory and confusing; the situation is worsened by studies that produce recommendations that are impossible to apply. This inconsistency is further promoted by intermittent drug supply and poor levels of training. At Chainama Hills College Hospital, although the staff are hard-working and willing, the high attrition rate and lack of training and mentorship compound the problems.

Zambia can do something about the poor evidence for the treatment of this most vulnerable (albeit aggressive) group of patients. After all, one of the first and most influential trials of healthcare came from a country ravaged by war, with insufficient funding for its health services to pay for the experimental treatments for everyone in need (Medical Research Council, 1948). The treatments used in Zambia in the management of aggression are not all evidence based. For example, use of haloperidol versus chlorpromazine for acute aggression caused by psychosis has been evaluated in one small trial. As these are the only two antipsychotic drugs that can be used for this purpose on the World Health Organization's List of Essential Medicines (World Health Organization, 2006b), the frequency of use worldwide is likely to be high. Brazil and India have already proven that relevant real-world trials of high methodological quality in this area are possible (Huf et al, 2002b; Alexander et at, 2004). Zambia could conduct similar or better trials to evaluate these medications and by doing so show the world how the river of evidence does not have to flow all in one direction.

There needs to be a clear career pathway for budding psychiatrists during their formative years, with more local training. Presently, many students take the often one-way journey out of the country to Kenya, Malawi or further afield. There are ongoing collaborative efforts to promote sharing of skills and knowledge transfer, both institutionally and nationally. Through some funding from the UK Department for International Development (DFID), working with the Tropical Health Education Trust (THET) and other organisations, the first ever Masters in Psychiatry course has been running in Zambia since May 2010. It is hoped that this will improve both the quality of care and the retention of psychiatrists.

Zambia is a land of immense beauty. The English language is widely spoken. Despite enormous poverty, people remain optimistic. In the 1980s, during a period of buoyant copper prices, significant improvements in healthcare occurred, including in the mental health services. Then copper prices collapsed and health provision fell. The situation is responsive and not entrenched. Things have improved before and can do again. This time, disengaged from the vagaries of the interests of big business, with modest support and the deep goodwill of local practitioners and organisers, improvements could and should last.

\section{References}

Alexander, J., Tharyan, P., Adams C., et al (2004) Rapid tranquillisation of violent or agitated patients in a psychiatric emergency setting. Pragmatic randomised trial of intramuscular lorazepam v. haloperidol plus promethazine. British Journal of Psychiatry, 185, 63-69.

Andrade, C. (2007) Rapid tranquillisation in emergency psychiatric settings. BMJ, 335, 835-836.

Atakan, Z. \& Davies, T. (1997) ABC of mental health. Mental health emergencies. BMJ, 314, 1740-1742.

Bourget, D., el-Guebaly, N. \& Atkinson, M. (2002) Assessing and managing violent patients. CPA Bulletin de l'APC, 25, 7 .

Huf, G., Coutinho, E. S., Fagundes, H. M., et al (2002a) Current practices in managing acutely disturbed patients at three hospitals in Rio de Janeiro, Brazil: a prevalence study. BMC Psychiatry, 2, 4 .

Huf, G., Coutinho, E. S. \& Adams, C. E. (2002b) TREC-Rio trial: a randomised controlled trial for rapid tranquillisation for agitated patients in emergency psychiatric rooms. BMC Psychiatry, 2, 11.

Medical Research Council (1948) Streptomycin treatment of pulmonary tuberculosis - a Medical Research Council investigation. $B M J$, ii, 769-82.

Pilowsky, L. S., Ring, H., Shine, P. J., et al (1992) Rapid tranquillisation. A survey of emergency prescribing in a general psychiatric hospital. British Journal of Psychiatry, 160, 831-835.

Sachdev, P. (1996) A critique of 'organic' and its proposed alternatives. Australian and New Zealand Journal of Psychiatry, 30 165-170.

Saxena, S., Sharan, P., Garrido, M., et al (2005) World Health Organization's Mental Health Atlas 2005: implications for policy development. World Psychiatry, 5, 179-184.

World Health Organization (2006a) Working Together for Health: The World Health Report 2006. WHO.

World Health Organization (2006b) The Selection and Use of Essential Medicines. Report of the WHO Expert Committee (Including the 14th Model List of Essential Medicines), Report No. 0512-3054. WHO. 